\title{
Laïcité, liberté de religion, État laïque
}

Les étapes de la laïcisation cubaine

\section{Jorge Ramírez Calzadilla}

Traducteur : Jean Hennequin

\section{(2) OpenEdition}

Journals

Édition électronique

URL : http://journals.openedition.org/assr/21278

DOI : $10.4000 /$ assr. 21278

ISSN : $1777-5825$

Éditeur

Éditions de l'EHESS

Édition imprimée

Date de publication : 1 juin 2009

Pagination : 157-182

ISBN : 978-2-7132-2216-0

ISSN : 0335-5985

\section{Référence électronique}

Jorge Ramírez Calzadilla, «Laïcité, liberté de religion, État laïque », Archives de sciences sociales des religions [En ligne], 146 | avril-juin 2009, mis en ligne le 01 juillet 2012, consulté le 30 avril 2019. URL : http://journals.openedition.org/assr/21278; DOI : 10.4000/assr.21278 


\section{Jorge Ramírez Calzadilla (†) ${ }^{1}$}

\section{Laïcité, liberté de religion, État laïque Les étapes de la laïcisation cubaine ${ }^{2}$}

Les rapports entre les organisations religieuses, l'État et le pouvoir civil, ainsi que le rôle de la religion et de ses institutions dans la vie publique au sein de la société, ont fait l'objet de vastes débats dans le monde moderne. On s'accorde généralement à affirmer que la modernité est née sous le signe de la laïcité, raison pour laquelle a été conçu, notamment en Occident, le projet de séparer les organisations religieuses du pouvoir politique et de confiner la religion à la sphère privée. Certaines positions extrêmes ont même prédit que, sous l'effet de la sécularisation propre à la modernité et sous le poids du progrès social, la religion finirait par disparaître, à un rythme plus ou moins rapide selon les cas. Dans ces conditions, la liberté religieuse, ou plus exactement la liberté de religion, devait s'exprimer en dehors de toute imposition, de tout favoritisme ou exclusion confessionnelle, de toute intolérance et de tout préjugé, en tant que manifestation des droits de l'homme, tels que ceux-ci trouvent leur expression dans la liberté de conscience et de pratique du culte préféré de chacun.

Toutefois, le monde actuel continue à être témoin de discriminations religieuses expressément déclarées, tantôt de façon évidente, tantôt sous des formes plus subtiles. Dans certains pays, une institution religieuse fait partie - de facto, sinon de jure - des structures du pouvoir politique. Rares sont les nations où le droit à la non-croyance fait l'objet d'une garantie juridique. La non-croyance tout comme la morale laïque et les conceptions athées sont au contraire prétexte à des préjugés d'intolérance, dans la mesure où elles sont considérées comme la preuve

1. Jorge Ramírez Calzadilla est décédé en 2007. Ce texte est publié à titre posthume. Traduction de Jean Hennequin.

2. Ce travail se fonde essentiellement sur une recherche inédite de J. Ramírez Calzadilla, Rafael Fernández, Sonia Jiménez, José Aróstegui et Liván Usallán (2003), Laicismo, libertad de religión y estado laico. Sus particularidades en la sociedad cubana, La Habana, Departamento de Estudios Sociorreligiosos, CIPS, Centro de Estudios sobre América e Instituto de Filosofía [Traduction de l'espagnol: Jean Hennequin]. Une première version en espagnol a été publiée dans R. Blancarte, (dir.), Los restos de la laicidad y la secularización en el mundo contemporáneo, México, El Colegio de México, 2008. La rédaction s'est autorisée à réduire légèrement ce texte, sans rien changer à son déroulement ni à sa problématique. 
d'un manque de principes et de valeurs éthiques élevées, ou encore d'une déloyauté citoyenne. Par ailleurs, l'expérience révèle que l'évolution de la religion, dans les divers rapports qu'elle entretient avec les autres phénomènes sociaux, avec des institutions telles que l'État, ainsi que dans les relations interpersonnelles, n'est pas allée dans le sens attendu : dans les faits, on assiste, en effet, à un renforcement des visées hégémoniques au sein de certains secteurs ecclésiastiques traditionnels.

Dans des conditions particulièrement agressives, les centres du pouvoir international tentent de manipuler le domaine religieux pour en faire un instrument d'opposition politique à des régimes et à des systèmes qui contredisent leurs intérêts hégémoniques, dans un monde unipolaire qui essaie d'imposer des formules néolibérales à partir d'une tendance à la globalisation, non seulement en matière économique, mais aussi politique et culturelle, y compris dans le domaine religieux.

D'un point de vue théorique, le concept de laïcité a fait l'objet de longs développements en science politique, mais aussi dans la littérature philosophique, juridique et sociologique. Son contenu et sa portée ont été largement débattus. Il a été particulièrement mis à l'honneur par le rationalisme, bien que celui-ci lui soit antérieur. De nos jours, il n'a rien perdu de son actualité. D’abord, du fait de la contradiction qui oppose les processus de sécularisation du monde moderne à la crise de la rationalité ayant servi de base à sa construction. Ensuite, en raison des énormes écarts qui séparent riches et pauvres, aussi bien d'un pays à l'autre qu'au sein de chaque société, ainsi que des agressions dont est victime l'environnement. L'abandon par les politiques néolibérales d'importantes masses dépossédées qui ne bénéficient en rien du marché, étant privées d'une capacité de consommation significative, favorise un essor religieux considérable à l'échelle mondiale ainsi qu'une demande corrélative d'utopies. Les religions deviennent une option alternative. Ainsi s'expliquent tout autant la persistance de fondamentalismes religieux que les violations de la liberté de religion dont il a été question ci-dessus.

Les différentes définitions qui, tout au long de l'histoire, ont été données des concepts de "laïcisme " ${ }^{3}$, de "laïcité " ou de "laïque ", sont fort éclairantes pour préciser le sens actuel de ces mots. Cependant, un tel examen nous conduirait bien au-delà des limites de ce travail. C'est pourquoi nous nous contenterons de proposer ici quelques définitions à des fins strictement pratiques et analyser certaines problématiques qui se posent de nos jours, en nous attachant tout particulièrement à la réalité cubaine qui constitue notre centre d'intérêt principal.

À Cuba, la laïcité, et en particulier l'État laïque, sont d'actualité. Non seulement en raison de l'orientation laïque de l'État et de la volonté d'impliquer la majorité

3. En espagnol: laicismo, terme qui n'a pas forcément une connotation négative, comme c'est parfois le cas du mot «laïcisme » en français. Bien qu'équivalent à celui de laicidad, le mot laicismo est plus courant à Cuba et en Espagne, et celui de laicidad dans le reste du monde hispanophone. Sauf pour le titre de l'article, dans le reste du texte nous avons suivi l'usage de l'auteur et traduit laicidad par "laïcité », et laicismo par "laïcisme » [Note du traducteur]. 
de la population dans la construction d'une société exempte d'intolérances, de préjugés et de discriminations débouchant sur des conflits stériles, mais aussi en raison du regain d'activité religieuse qui a accompagné la crise des années quatre-vingt-dix, connue sous le nom de "période spéciale » : une période au cours de laquelle les espaces sociaux des institutions religieuses se sont élargis, leur rayonnement et leurs demandes ont été reconsidérés, tandis que, depuis l'extérieur, on tentait d'introduire des tendances religieuses non traditionnelles nettement individualistes qui encourageaient des attitudes de désengagement social.

\section{Le concept de laïcité : déterminations et problématiques actuelles}

La pensée bourgeoise et, notamment, le rationalisme dès les XVII et XVIII siècles ont mis l'accent sur la séparation des sphères politique et religieuse et érigé l'État laïque au rang d'idéal. Divers pays ont entériné ces aspirations dans le domaine juridique et dans la pratique, tandis que d'autres en sont restés au stade de simples tentatives : quoi qu'il en soit, il s'est agi dans tous les cas de rechercher un modèle d'organisation macro-social dans lequel le domaine laïque a la primauté et se trouve séparé de l'Église, tandis que le domaine religieux, auquel est dévolu un rôle secondaire, se trouve réduit au rang de conviction et relégué à la vie ecclésiastique.

Le rationalisme, ainsi que les Lumières en tant que modalité de ce rationalisme, ont été une réaction bourgeoise face à l'ordre féodal, à l'État monarchique - non dénué d'une certaine connotation théocratique - et face au pouvoir considérable de l'Église et à l'emprise de la religion sur la vie publique.

La construction de la société moderne - bourgeoise - sur des bases rationnelles, est due objectivement au fait que la reproduction de la société capitaliste s'appuie sur des mécanismes basés sur des lois économiques, des facteurs sociaux et politiques, selon une logique fondée sur des rapports matériels, de sorte que le «métasocial »- pour reprendre la terminologie de F. Houtart ${ }^{4}$ - et, par conséquent, le surnaturel ne constituent pas des ressources indispensables, même si la religion est utilisée en tant que légitimation complémentaire. Dans ces conditions, la vie sociale suit une évolution essentiellement laïque et l'État ainsi que l'ordre social ne se fondent plus sur une légitimation nécessairement religieuse. Le pouvoir et les fonctions publiques furent ainsi désacralisés, la tendance étant à remplacer la religion par la rationalité. Dans les faits, comme conséquence du rationalisme, l'Église fut détrônée dans divers pays occidentaux par le pouvoir politique, même si, de toute évidence, la religion ne disparut pas.

L'usage a associé les adjectifs « laïque ", "séculier » et "athée " pour qualifier l’État, notamment «l'État laïque ». Dans le domaine de la pratique politique, il

4. Parmi l'abondante production de François Houtart, un ouvrage est particulièrement recommandé pour une approche globale de la religion: Sociologie de la religion, Managua, Nicarao, La Havane, CEA, 1992. 
en va de même des concepts de "sécularisation " ou de "désacralisation ", ainsi que de l'antinomie entre le "public " et le "privé ", voire de celui de "société civile». On peut donc les considérer comme formant une famille de concepts reliés les uns aux autres, surtout de par leurs implications pratiques, plus que comme un système catégoriel à proprement parler.

En principe, et sans poursuivre d'autre but que celui de nous rapprocher d'une utilité pratique, nous pourrions nous accorder à donner de ces concepts les définitions minimales que voici :

Le mot "laique » se réfère à un domaine à caractère politique, culturel et éthique, et qui se différencie du domaine proprement religieux, sans qu'il y ait contradiction ni exclusion entre les deux. Ce qui est laïque, c'est ce qui est autre par rapport au religieux, ce qui n'est pas fondé sur l'acceptation du surnaturel, sans pour autant le nier nécessairement. Par conséquent, le mot « laïque » n'est pas exactement synonyme de non-croyant, et moins encore d'antireligieux.

L'adjectif « séculier » se réfère aux choses terrestres, mondaines ou appartenant au monde (ce qui, d'un point de vue sociologique, n'implique aucune connotation péjorative, contrairement à certaines conceptions théologiques dichotomiques pour lesquelles le mondain relève essentiellement du péché). Le séculier s'oppose au sacré, bien qu'il puisse également l'inclure, dans la mesure où il est conçu par certaines théories religieuses comme le domaine dans lequel est à l'œuvre le surnaturel.

«Athée » signifie étymologiquement « sans dieu ». Il s'agit donc de la conception dans laquelle est exclue l'acceptation de Dieu ou de dieux. Toutefois, cet adjectif n'est pas synonyme de non-croyant, dans la mesure où la pratique sociale nous montre qu'il existe certaines croyances religieuses qui ne se structurent pas sous forme de figure dotée des attributs suprêmes qui, historiquement, sont attribués à la divinité. Tel est le cas, à Cuba, de la religiosité populaire dans laquelle il existe de multiples croyances qui ne se cristallisent pas sous forme de divinité et dans lesquelles le surnaturel n'est pas même personnifié 5 .

La « sécularisation », « désacralisation » ou « laïcisation », est le processus qui a accompagné la modernité et qui tend à réduire le rôle de la religion au sein de la vie sociale, ainsi que sa capacité d'intervention et d'influence sur les rapports sociaux. Ce processus repose sur une base objective, à savoir les facteurs sociaux qui le déterminent, et s'exprime à travers des théories et des comportements. Au niveau de la conscience et de la pratique sociale, le laïcisme prend le dessus sur le religieux.

5. Voir l'ouvrage collectif intitulé La conciencia religiosa. Características y formas de manifestarse en la sociedad cubana actual, La Habana, Departamento de Estudios Sociorreligiosos, CIPS, 1993. 
Les frontières entre "le public » et «le privé » sont fort imprécises. Ces deux concepts se réfèrent à des phénomènes, des processus, des conceptions, des activités, des attributs et des droits en rapport les uns avec les autres, voire se complétant dans certains cas ou, au contraire, s'opposant les uns aux autres. Le contenu de ces deux domaines se trouve relativisé par divers facteurs sociaux et, bien souvent aussi, par des conjonctures économiques, politiques ou idéologiques.

Pour la plupart des Églises chrétiennes, l'adjectif « laïque », outre son sens de non religieux, de séculier, s'applique aux croyants qui n’ont pas été consacrés, qui ne font pas partie du clergé ou du pastorat. Dans ce même sens, l'Église catholique emploie le terme de "séculier » : c'est pourquoi on parle indistinctement de mouvements ou d'organisations laiqques ou séculières pour se référer à des actions ou à des associations engagées vis-à-vis de l'Église et placées sous le contrôle plus ou moins strict du clergé. Ceci est source de confusion pour qui n'emploie le terme de "laïque » que pour se référer, précisément, aux membres d'une Église.

Par ailleurs, l'Église catholique qualifie aussi de "séculière » la partie du clergé faisant partie du "siècle » (saeculum), c'est-à-dire du monde, par opposition au clergé "régulier", qui s'organise sous forme de communautés soumises à une règle (regula) ou à un règlement.

Dans la vie sociale, le terme « laïque » fait référence à une conception du monde et un rapport au monde auxquels est étrangère toute vision religieuse, aussi bien au niveau individuel et groupal que social. On peut donc parler d'une philosophie laïque, d'une éthique laique, d'une politique laïque, d'un droit laïque, d'institutions laïques, d'un État laïque, de rapports laïques et d'une pratique laïque.

En résumé, le laïcisme, outre les diverses interprétations auxquelles il a donné lieu, se trouve déterminé, dans son expression au sein de la pratique sociale, par un ensemble de facteurs objectifs et subjectifs qui nous obligent à l'examiner dans son contexte. Il se manifeste essentiellement sous deux formes d'une singulière complexité et qui renferment un important potentiel conflictuel dans le monde contemporain : la liberté religieuse ou, plus exactement, la liberté de religion, et l’État laïque.

\section{La liberté de religion}

L'un des aspects qui, d'un point de vue historique, a donné à la religion une dimension problématique, est le droit de pratiquer l'une ou l'autre de ses formes ou de n'en pratiquer aucune, ce à quoi sont liées, par opposition, toutes sortes d'intolérances, de discriminations, d'hégémonies. La religion a été présente dans bien des conflits violents, qu'elle en ait été la cause principale ou la cause apparente. Le monde contemporain n'est pas exempt de problèmes de cette nature.

Ce n'est qu'en l'envisageant au sein des réseaux de relations multiples dans lesquels elle s'inscrit, et en tenant compte du contexte sociohistorique, que l'on 
peut parvenir à une compréhension approfondie de la liberté de religion. C'est pourquoi certains analystes, tout en soulignant l'universalité et, par conséquent, la transcendance des droits de l'homme - parmi lesquels se trouve la liberté de religion - face à la variabilité des déterminations, posent, sans toutefois céder aux affirmations du relativisme culturel, que ceci n'implique nullement de méconnaître «le conditionnement et la particularité historiques ${ }^{6}$. La liberté de religion est modelée par des circonstances spécifiques qui la limitent ou lui permettent de se manifester plus ouvertement, et décident de son contenu et de son caractère.

Par ailleurs, la liberté de religion doit être traitée de façon objective. On ne saurait ni la sous-estimer, ce qui peut conduire à la subordonner indûment à d'autres facteurs sociaux et à porter ainsi préjudice aux croyants, ni la surestimer au point d'en faire la «base de toutes les autres libertés » ${ }^{7}$, comme le proclame souvent le discours des dirigeants religieux.

Aux termes de la Déclaration Universelle des Droits de l'Homme, approuvée en 1948 par l'Assemblée Générale des Nations Unies, la religion ne saurait constituer une caractéristique distinctive permettant de jouir d'un droit quelconque ; en outre, cette Déclaration proclame la liberté d'adopter, de changer et de manifester sa religion de manière individuelle, collective, privée et publique ${ }^{8}$. La liberté religieuse a reçu de nombreuses interprétations et applications avant et après cette Déclaration ${ }^{9}$, en fonction des conjonctures propres aux différents pays.

La liberté de religion constitue, d'une certaine manière, l'expression des rapports Église-État et, plus encore, des rapports religion-société ; par conséquent, outre le fait qu'elle s'exprime à travers des théories et des aspirations, autant à l'intérieur de systèmes de pensée que de programmes politiques, elle a son existence sociale réelle dans la pratique de rapports, de comportements et de potentialités, qui ne sont d'ailleurs pas toujours en accord avec ce qui est proclamé. Elle est non seulement synonyme de pluralité religieuse, comme le défend la libre pensée, mais aussi de possibilité, pour les sujets et les groupes, de choisir une forme de religion ou de n'en choisir aucune, en dehors de toute contrainte ou de toute implication négative.

6. J. Donelly, Derechos Humanos Universales. En teoría y en la práctica, México, Éd. Gernika SA, 1994, p. 28.

7. Message du Pape Jean-Paul II à Kurt Waldheim, secrétaire général de l'ONU, le 2 décembre 1978, à l'occasion du XX $\mathrm{XX}^{\mathrm{e}}$ anniversaire de la Déclaration Universelle des Droits de l'Homme (La libertad religiosa, textos de Juan Pablo II, Ciudad del Vaticano, Comisión Pontificia Justitia et Pax, 1980, pp. 11-13). Cette idée est reprise dans un ouvrage postérieur qui résume sa pensée : Cruzando el umbral de la esperanza, Barcelona, Ed. Plaza y Janés, 1994.

8. C'est en ces termes qu'il est fait référence à la religion dans le Préambule, ainsi que dans les articles 2, 10 et 18 de la Déclaration Universelle des Droits de l'Homme.

9. J. Ramírez Calzadilla, «Libertad de conciencia y religión en Cuba », Revista Cubana de Ciencias Sociales, 25, La Habana, 1991, pp. 133-156. 


\section{L'État laïque, sa conceptualisation}

L'expression concrète la plus évidente du laïcisme se trouve peut-être dans l'application juridique et dans la pratique étatique. En matière de religion, l'État doit être régi par la loi, c'est-à-dire aussi bien par les articles constitutionnels que par certaines réglementations spécifiques, recueillies dans ce que l'on a coutume d'appeler, à Cuba, de façon d'ailleurs assez curieuse, la Loi des Cultes - celle-ci n'ayant pas pour objet les activités rituelles, mais le traitement à réserver aux associations religieuses, leurs possibilités d'action et leurs droits face à l'État. C'est en fonction de la nature de sa législation, mais plus encore en fonction de sa manière d'agir dans la pratique, que l'on pourra considérer tel ou tel État comme laïque ou comme confessionnel.

Neutralité en matière religieuse, séparation entre l'Église et l'État, établissement de l'éducation publique laïque, traitement égalitaire des différentes religions : l'État laïque ne se réduit pas à l'une ou l'autre de ces dispositions prises isolément. Au contraire, l'État laïque suppose l'ensemble de ces dispositions doublé d'une stratégie et d'une volonté politique : «... l'État laïque n'est pas l'État confessionnel... l'État est laïque lorsqu'il n'a plus besoin de la religion comme élément d'intégration sociale ou comme ciment de l'unité nationale » ${ }^{10}$. Un État laïque fait référence à un certain nombre de principes, parmi lesquels on peut mentionner les suivants : - séparation entre l'Église (y compris toute organisation religieuse, qu'elle possède ou non une structure ecclésiastique) et l'État, en tant qu'institutions de nature différente, disposant chacune d'une autonomie tant économique que de fonctionnement ;

- traitement égalitaire des religions existantes de la part de l’État (pas de religion privilégiée, pas de religion persécutée), à condition qu'elles se conforment à la loi et à la morale et ne portent pas atteinte à la santé ni à l'intégrité des personnes (cette précision pourrait sembler superflue si l'on s'en tenait au concept éthique de "religion " actuellement en vigueur ; cependant, on ne saurait oublier qu'il a existé et continue d'exister certaines tendances religieuses - ou plus exactement peut-être, pseudo-religieuses - auxquelles ces dispositions sont applicables); - exercice garanti de la liberté de religion (c'est-à-dire aussi bien le droit à avoir des croyances religieuses, à changer de croyance et à pratiquer un culte, qu'à n'en avoir aucune et à ne pratiquer aucun culte). L'État laïque représente autant les croyants d'une religion ou d'une autre, que les non-croyants ;

- l’État ne doit pas encourager la religion, ni aucun type de religion en particulier (le prosélytisme incombant à chacune des différentes organisations religieuses); toutefois, il ne doit ni la persécuter ni la discriminer ;

10. Roberto Blancarte, «La laicidad mexicana; retos y perspectivas », communication présentée lors du colloque Laicidad y valores en un estado democrático, México, El Colegio de México, 6 avril 2000. 
- les organisations religieuses sont légalement enregistrées. Chacune a le droit d'exercer les activités qui lui sont propres et qui fondent sa raison d'être ;

- laïcité de la morale et de l'éducation officiellement professées et diffusées, ce qui ne signifie nullement que soit exclue la morale religieuse, ni la possibilité de recevoir l'instruction religieuse que les organisations enregistrées sont susceptibles d'assurer par leurs propres moyens;

- interdiction d'entraver l'exercice du culte religieux d'une organisation légalement enregistrée et d'intervenir dans le déroulement de celui-ci, ainsi que dans les structures organisationnelles de ces organisations.

Ces principes comportent aussi bien des droits que des obligations, tant pour l'État que pour les organisations religieuses. On a souvent tendance à oublier cette dualité et à envisager les problèmes de façon unilatérale.

\section{Laïcisme, liberté de religion et État laïque à Cuba}

À Cuba, le laïcisme a connu une évolution non exempte de quelques particularités qui, d'une certaine manière, la différencient de la situation qui prévaut dans les autres pays d'Amérique latine. Bien entendu, ceci n'implique nullement que la culture cubaine ne participe pas de l'identité latino-américaine ni, évidemment, caribéenne, dans la mesure où les caractéristiques propres à Cuba présentent une double appartenance, à la fois au monde latino-américain et au monde caribéen. N'oublions pas que l'identité implique la différence.

Dès la naissance de la nation cubaine, le développement des idées dans ce pays a été soumis à la forte influence de la libre pensée, du laïcisme, de l'antidogmatisme et de l'anticléricalisme ; cependant, le développement de la pensée à Cuba n'a pas été soumis autant que dans d'autres pays de la région à l'influence antireligieuse, comme il ressort de la production intellectuelle cubaine, ainsi que de la législation concernant la religion, notamment des différentes Constitutions.

À l'égard de la religion dominante, cette idéologie critique s'est exprimée en termes d'opposition : a- au dogmatisme philosophique et à son application dans l'enseignement. Cette opposition se cristallisa autour de la figure de Félix Varela qui très tôt réunit en lui l'idéal indépendantiste et l'idéal antiscolastique ; b- aux interprétations antiscientifiques initialement sous l'influence du darwinisme et du positivisme ; c- à la ligne d'action politique de l'Église officielle et à l'hégémonie spirituelle et politique que celle-ci exerça pendant longtemps, opposition qui se traduisit par l'anticléricalisme, le déisme, la libre pensée, voire, dans une moindre mesure, l'athéisme.

Félix Varela élabora et mit en pratique ses théories précocement, ce qui fit de lui et de José Agustín Caballero les fondateurs de la pensée nationaliste qui, d'une manière ou de l'autre, influença les générations postérieures. Il est intéressant de noter que, paradoxalement, tous deux étaient prêtres et n'avaient d'ailleurs 
pas bonne presse auprès du clergé espagnol, notamment auprès de leurs supérieurs hiérarchiques, comme il apparaît dans les contestations formulées à l'encontre de l'évêque Espada qui les protégeait et les encourageait. S'ils ne pouvaient être, et ne furent pas, anticléricaux et encore moins athées, leur antidogmatisme et leur idéal de liberté jetèrent les bases de l'œuvre la plus solide que construiront par la suite leurs partisans.

Les penseurs cubains qui ont laissé les traces les plus profondes dans la conscience nationale du pays se situent dans le sillage de cette conscience critique : outre Martí, le plus étudié et le plus emblématique, citons Felipe Poey, Antonio Mestre, Julio Sanguily, Luz y Caballero, au XIX ${ }^{\mathrm{e}}$ siècle; puis Varona et bien d'autres encore vivants au $\mathrm{XX}^{\mathrm{e}}$ siècle, voire au début du XXI ${ }^{\mathrm{e}}$ siècle, tels Rubén Martínez Villena, Julio Antonio Mella, Juan Marinello, Fernando Ortiz, Emilio Roig de Leuchsering et d'autres représentants de la Génération des Années Trente ; sans oublier ceux de la Génération du Centenaire, sous la conduite de Fidel Castro, ni Che Guevara qui, s'il ne se prononça guère sur la question religieuse, affirma sa conviction que la révolution latino-américaine ne deviendrait possible que lorsque les chrétiens seraient capables de s'engager inconditionnellement dans la lutte.

Cette façon de penser ne s'est pas seulement manifestée en théorie, mais également dans la pratique politique et juridique. Tel fut le cas, notamment, des Constitutions de la République en Armes qui jetèrent les bases d'une tradition séparatiste, recueillie par les Constitutions suivantes et en vertu de laquelle aucune religion ne doit être privilégiée par rapport aux autres : ce principe a été défini à Cuba avant de l'être dans d'autres pays du continent qui s'affranchirent du colonialisme espagnol, sans avoir signé de concordat ou d'accord particulier avec le Vatican ou avec une autre Église, une fois révolu le Patronato Regio de l'époque coloniale; actuellement encore, Cuba est l'une des rares nations où la Constitution reconnaît le droit à la non-croyance et où il n'existe aucune interdiction à ce que des ministres du culte exercent des fonctions publiques au sein de l'État, contrairement à ce que prévoient d'autres Constitutions de plusieurs pays.

La libre pensée et l'anticléricalisme ne supposent pas nécessairement l'athéisme. Outre l'acceptation de la pluralité religieuse, ce qui est commun aux divers penseurs cubains, c'est la critique radicale du rôle politique joué à Cuba par la religion, et par l'Église catholique en particulier. Chez ces penseurs, on constate des points de vue variés qui permettent de porter des appréciations selon différentes perspectives. Sanguily, par exemple, déclara lors des séances de l'Assemblée constituante de 1901: «A Cuba, la religion et l'ignorance sont toujours allées main dans la main et l'Église, de par sa conduite, s'est éloignée du peuple, contribuant ainsi à ce que beaucoup deviennent irréligieux » ${ }^{11}$. Pour Martí, "... l'Église a toujours

11. Foner Phillips, "La guerra hispano-cubano-americana y el surgimiento del imperialismo yanqui ", vol. I, p. 234. 
été l'alliée par excellence des puissants ${ }^{12}$; et toujours selon lui - ce qui ne doit pas nous induire à le considérer erronément comme un athée -, «...ce sont les hommes qui inventent les dieux à leur image » 13 . Varona, pour sa part, révèle son agnosticisme à travers des phrases comme celle-ci : « À l'ombre de la religion, et favorisées par elle, ont fleuri les pires aberrations » ${ }^{14}$. Plus tard, analysant la conduite du clergé durant la conquête et la colonisation, Fernando Ortiz le qualifia en général de belliqueux, intransigeant, ignorant, pauvre d'esprit, égoïste ${ }^{15}$. Une organisation telle que l'Agrupación Católica Universitaria (ACU), qu'on ne saurait soupçonner de parti pris défavorable à l'Église, recueillit les résultats d'une enquête nationale effectuée en 1953, selon laquelle 39,4\% des enquêtés n'adressèrent, au total, pas moins de deux mille sept cent soixante objections au clergé et à l'Église ${ }^{16}$. On peut donc en conclure qu'il s'agit là d'un anticléricalisme, sinon massif, tout au moins assez généralisé.

\section{Les premières Constitutions de Cuba: une réaction au Patronato Regio}

L'histoire cubaine doit être conçue comme étant composée de trois phases principales : l'étape coloniale (conquête et colonisation espagnole), l'étape républicaine néocoloniale (1902-1959) et l'étape révolutionnaire, à partir de cette dernière date. Pour chacune d'entre elles, les conjonctures qui marquèrent la sphère religieuse sont différentes. Tout au long de cette évolution sociohistorique, la religion a acquis à Cuba trois caractéristiques principales ${ }^{17}$. En premier lieu, l'ensemble des manifestations religieuses concrètes, qui forment ce que l'on pourrait dénommer le cadre religieux à Cuba, est divers, hétérogène, voire contradictoire. Plus qu'au nombre de ses différentes manifestations, sa complexité est due aux origines variées des manifestations religieuses en présence.

En deuxième lieu, pour les raisons les plus diverses, aucune expression religieuse organisée ne l'a emporté sur les autres, de manière à conférer à la religiosité cubaine un caractère vraiment typique. Elle s'exprime sous des formes relativement indépendantes de l'orthodoxie de systèmes religieux spécifiques. Cette religiosité

12. José Martí, «Obras completas ", p. 393.

13. Idem, tome XVIII, p. 30.

14. Enrique José Varona, "Trabajo sobre educación y enseñanza », Comisión Nacional de Cuba de la UNESCO, La Havane, 1961.

15. Fernando Ortiz, Los negros brujos, Madrid, Editorial América, 1979, pp. 21 et 29.

16. ACU, «Encuesta Nacional sobre Sentimientos Religiosos », La Habana, Buró de Información de la Agrupación Católica Universitaria, 1954.

17. Pour une étude approfondie du cadre religieux, de la religiosité prédominante et de la signification sociopolitique de la religion à Cuba, voir les travaux du DESR, par exemple: Ouvrages collectifs: La religión en la cultura, La Habana, Editorial Academia, 1990; La conciencia religiosa. Características y formas de manifestarse en la sociedad cubana actual, La Habana, 1993 (inédit); J. Ramírez, Religión y relaciones sociales, La Habana, Editorial Academia, 2000. 
s'inscrit dans le cadre de la vie quotidienne et peut être qualifiée de populaire. Elle est, à l'instar de la culture cubaine, une synthèse originale de diverses composantes.

En troisième lieu, dans aucun des systèmes socioéconomiques implantés, l'élément métasocial que représente la religion n'a constitué la base de la reproduction sociale. Pour cette raison, la religion à Cuba n'a pas atteint des niveaux très élevés de signification sociale, tout au moins en comparaison avec d'autres pays latino-américains.

Sous le système colonial espagnol, s'implantèrent à Cuba le catholicisme, en tant que religion dominante et pratiquement exclusive, diverses religions africaines, qui évoluèrent vers des formes nouvelles sous l'impulsion de la société cubaine, puis, vers la fin du XIX ${ }^{\mathrm{e}}$ siècle, le spiritisme et le protestantisme, en provenance des États-Unis. La culture et la religiosité anciennement développées à Cuba ne revêtirent jamais une grande importance, contrairement à ce qui se produisit dans d'autres régions latino-américaines, où les cultures inca et méso-américaine possèdent aujourd'hui encore une grande vitalité. Au cours de cette phase, la liberté religieuse était pratiquement inconnue, l'Église catholique entretenant un rapport organique avec la Couronne espagnole.

Le Patronato Regio, instrument chargé de réguler les rapports entre l'Église et l'État, plaçait de fait l'institution ecclésiastique sous la dépendance des intérêts politiques, mais permettait néanmoins à celle-ci de bénéficier d'un important pouvoir hégémonique. Dans ces conditions, il était impossible pour les Églises protestantes de s'installer à Cuba. Pour leur part, le judaïsme et l'islam avaient été exclus de la métropole, tandis que les religions africaines étaient soumises à un processus visant à les éliminer : les partisans de ces religions furent évangélisés par la force, bien que l'on n'ait pas recouru, dans ce cas, à l'extermination ethnique des Africains et de leurs descendants; ceux-ci parvinrent néanmoins à préserver leur culture, incrustée au sein de la "cubanité ", non seulement grâce à un processus de résistance, mais surtout en raison de l'incapacité du système à assurer leur conversion réelle au catholicisme ${ }^{18}$. D'où l'importante présence africaine au sein de la religiosité cubaine.

L'instrumentalisation de la religion en fonction des intérêts coloniaux - instrumentalisation qui revêtit des formes plus violentes au cours des luttes d'indépendance - donna naissance, à l'intérieur de la pensée cubaine émergeante et dans la pratique de la «cubanité », à l'anticléricalisme qui marqua la société cubaine, quoique ses manifestations n'aient pas été aussi extrêmes que dans d'autres régions. Ce fait, joint aux influences de la libre pensée, marqua les Constitutions élaborées durant la période de la République en Armes.

18. J. Ramírez Calzadilla, «Persistencia religiosa de la cultura africana en las condiciones cubanas », Revista Catauro, II-3, enero-junio, [La Havane, Fundación Fernando Ortiz,] 2001, pp. 106-127. 
La Constitution de la République de Cuba, approuvée le 10 avril 1869 à Guáimaro, Camagüey, en pleine guerre d'indépendance, disposait dans son article 28: "La Chambre ne saurait aller à l'encontre des libertés de culte, de presse, de réunion pacifique, d'enseignement ni de pétition, ni d'aucun droit inaliénable du peuple ». Celle de Jimaguayú (une autre commune de Camagüey), approuvée le 16 septembre 1895, n'aborde pas le thème de la religion. Quant à celle de Yaya (région de Camagüey), ratifiée le 29 novembre 1897, elle déclare dans son article $6:$ : Les Cubains ainsi que les étrangers jouiront de la protection de la loi en ce qui concerne leurs opinions religieuses et l'exercice de leur propre culte, dans la mesure où ceux-ci ne s'opposent pas à la morale publique». L'article 13, pour sa part, stipule que «tous les Cubains ont le droit d'exprimer librement leurs idées, de se réunir et de s'associer en vue de n'importe quel but licite de la vie".

Comme on le voit, aucune de ces Constitutions ne consacre l'existence d'une religion officielle ou privilégiée; deux d'entre elles garantissent explicitement la liberté de culte, et une la liberté de croyances - ces dernières étant désignées sous le nom d'" opinions religieuses ". Le fait que l'une de ces Constitutions ne fasse pas allusion à la question religieuse était à l'époque synonyme de neutralité. En tant qu'expression de la libre pensée, ces Constitutions établissent un pluralisme religieux face au Patronato Regio, ainsi qu'à l'hégémonie et à l'exclusivité du catholicisme consacrées par cet instrument. Se trouvait ainsi inaugurée une tradition qui sera conservée et amplifiée par la suite.

\section{L'étape républicaine néocoloniale}

La phase républicaine fut précédée d'une étape intermédiaire, une fois achevée la domination espagnole, celle de l'intervention américaine (1898-1902) qui favorisa l'implantation des Églises protestantes sous le contrôle de leurs Conseils Missionnaires et expulsa les premiers missionnaires cubains, dont certains participèrent aux luttes d'indépendance. À partir de ce moment, et tout au long de cette étape, les Églises protestantes apportèrent, à des degrés divers, leur soutien aux États-Unis ; en ce sens, Werren A. Candler, évêque méthodiste de Géorgie, alla jusqu’à déclarer que l'Église devait compléter le travail « du soldat et du marin ${ }^{19}$.

Le panorama religieux ne cessa de se diversifier, tandis qu'était mise en place une pratique obéissant aux critères de la libre pensée, tels que ceux-ci étaient garantis par la Constitution, et que s'établissaient des rapports de concurrence entre les différentes organisations religieuses. Toutefois, l'Église catholique parvint peu à peu à recouvrer sa position privilégiée, tandis que des discriminations

19. Rafael Cepeda, (ed.), La herencia misionera en Cuba, San José de Costa Rica, Departamento Ecuménico de Investigaciones, 1986, p. 45. 
étaient exercées à l'encontre des religions d'origine africaine et du spiritisme, assez répandus parmi le peuple, et que les non-croyants étaient victimes de divers préjugés.

Les États-Unis expérimentèrent à Cuba un système néocolonial qui eut pour résultat de frustrer les idéaux de l'indépendance. En matière religieuse, ceci se traduisit par trois phénomènes principaux : l'établissement d'une pratique inspirée de la libre pensée et de la multiplicité religieuse, ce qui coïncidait avec les aspirations les plus avancées du mouvement laïque; la faveur accordée aux Églises protestantes, conformément aux intérêts idéologiques américains; le maintien d'une certaine légitimation de l'Église catholique, en accord avec certains gestes favorables de la part du Vatican et avec le fait que cette institution était, malgré sa conduite précédente, celle qui conservait le plus d'influence sur la société. L'Église catholique était, en fait, la seule institution ayant préservé sa structure après une longue guerre dévastatrice.

En 1901 fut promulguée la première Constitution républicaine qui, après avoir invoqué «la faveur de Dieu ", établissait dans son article 26, alinéa 4 : "Chacun est libre de professer la religion et le culte de son choix, sans aucune autre limitation que le respect de la morale chrétienne et de l'ordre public »; et plus loin : "L'Église sera séparée de l'État, ce dernier ne pouvant en aucun cas subventionner aucun culte $"$. L'article 31 proclame le caractère obligatoire de l'enseignement primaire, ainsi que de celui des Arts et Métiers, tous deux étant à la charge de l'État. En 1898, le gouverneur américain Leonard Wood avait proposé une Constitution provisoire qui consacrait la liberté de culte, quoique uniquement pour les chrétiens.

Quant au débat que suscita la proposition d'inclure le nom de Dieu dans le préambule de la Constitution, le Dr. Fernández Bulté a signalé que celui-ci alla bien au-delà de cette invocation elle-même. Ce juriste rappelle que Salvador Cisneros Betancourt en profita pour faire une déclaration d'athéisme et censurer certaines autorités ecclésiastiques comme Sbarreti, qui venait à peine d'être nommé évêque de La Havane. Il s'opposa catégoriquement à ce que le nom de Dieu soit mentionné dans la Constitution ou figure dans la vie politique du pays. D’autres délégués, au contraire, approuvèrent cette proposition, notamment González Llorente qui proclama son adhésion au catholicisme. Ce fut Manuel Sanguily qui contribua à trancher le débat, non pas grâce à une définition religieuse, mais en s'exprimant en ces termes : «Dieu est, en fin de compte, le symbole du bien qui se réalise avec nous, contre nous, malgré nous, maintenant, dans le passé et dans l'avenir " et en proposant, dit Fernández Bulté, que "ce symbole soit invoqué comme étant à la mesure de l'espérance et des intentions bienveillantes du peuple cubain ${ }^{20}$.

20. Dr. Julio Fernández Bulté : communication présentée dans le cadre des Résultats de recherche sur la laïcité, Conseil Scientifique du CIPS, 13 novembre 2003. 
Ainsi, la Grande Charte qui marquait la naissance de la république conservait la tradition de libre pensée qui avait déjà caractérisé les Constitutions de la République en Armes durant les guerres d'indépendance. Il est significatif que Cuba, bien qu'ayant accédé tardivement à l'indépendance, ait décrété, avant bien d'autres pays du continent américain, la séparation entre l'Église et l'État à laquelle aspiraient la pensée moderne ainsi que la bourgeoisie libérale, mais qui suscitait les plus vives réticences de la part de l'Église. Il s'agissait sans conteste d'un texte fort avancé pour l'époque, même s'il présentait l'inconvénient d'imposer la morale chrétienne aux non-chrétiens et aux non-croyants, auxquels il ne reconnaissait d'ailleurs pas le droit d'être tels.

En 1933 et 1935 furent promulgués tour à tour des statuts à l'intention du gouvernement provisoire, ne contenant aucune allusion à la religion, et une loi constitutionnelle qui, en matière de religion, se contentait de reprendre ce qui avait été établi par l'article 26 de la Constitution de 1901. En 1940, fut promulguée la seconde Constitution républicaine qui, comme la première, invoquait la «faveur de Dieu ", el favor de Dios, ce qui, cette fois, donna lieu à une importante controverse, en raison de l'opposition que suscita une telle invocation parmi un certain nombre de constituants progressistes - et établissait, de même que la première, la liberté de profession pour toutes les religions, "sans autre limitation que le respect de la morale chrétienne et de l'ordre public». Nouveauté importante à signaler, l'article 43 établissait que "seul est valable le mariage autorisé par des fonctionnaires légalement habilités à cet effet ", ce qui revenait à enlever toute validité officielle au mariage religieux ; pour sa part, l'article 55 établissait que l'enseignement officiel était laïque, mesure que l'on peut qualifier d'avancée dans le contexte latino-américain de l'époque, bien que les établissements éducatifs privés aient eu la liberté d'enseigner n'importe quelle religion.

Ce sont exactement les mêmes dispositions qui, en tant que manœuvre destinée à pallier le caractère anticonstitutionnel d'un régime imposé de facto, furent adoptées par la loi constitutionnelle de la République de Cuba, approuvée le 4 avril 1952.

\section{Étape révolutionnaire}

Après une vingtaine d'années de vie républicaine, l'Église catholique parvint à résoudre les contradictions qui avaient entravé jusqu'alors ses relations avec la bourgeoisie nationale. Violant les principes constitutionnels, l'institution ecclésiastique parvint à nouveau, quoique dans une moindre mesure que lorsqu'elle jouissait de son statut colonial, à nouer d'étroits rapports avec le pouvoir politique, ce qui lui permit de jouir de certains privilèges dépassant ceux des autres organisations religieuses. Parallèlement, elle se mit en devoir d'offrir d'elle-même une image plus «cubanisée ». Les relations diplomatiques avec le Vatican, établies vers le milieu de cette période, contribuèrent à renforcer son rôle grâce à un soutien 
international plus puissant que celui dont disposaient les Églises protestantes à travers leurs «Églises mères ».

Le protestantisme prospéra tout au long de cette période, reproduisant la diversité propre à la société américaine. Certains documents attestent l'inquiétude permanente que suscitait parmi les représentants de ces Églises le pouvoir hégémonique de l'Église catholique, tel que celui-ci se manifestait dans les rapports privilégiés lui permettant de prendre part, de facto sinon de jure, au pouvoir politique. En outre, les lois obligeaient ces institutions à procéder à leur enregistrement officiel, obligation dont l'Église catholique se trouvait, de manière totalement inconséquente, exonérée. C'est au cours de ces années que fut créé le Concile Cubain des Églises, qui par la suite changea plusieurs fois de nom, pour porter maintenant celui de Conseil des Églises de Cuba. Son intention première était de constituer un front uni face au catholicisme, ce qui ne l'empêcha pourtant pas de poursuivre des objectifs d'unité religieuse, ni de se transformer plus tard en un mouvement acuménique doté d'un important rayonnement social.

Face au peu d'intérêt que leur portait l'État, les Églises chrétiennes se consacrèrent à des tâches d'éducation et d'assistance sociale. Les collèges privés, auxquels étaient affectés la plupart des membres du clergé catholique, représentaient une importante source de revenus pour ces Églises. Les fonctions de cette nature étaient tenues en grande estime par les institutions religieuses, à tel point qu'elles constituaient la principale raison d'être de nombre d'entre elles. Dans cette perspective, on comprend que la réduction, voire la perte de ces fonctions que les Églises accuseront par la suite, donnera lieu à bien des conflits avec l'État. L'État républicain se désintéressa des autres expressions religieuses, si bien que le spiritisme, et surtout les religions d'origine africaine telles que la santería, le palo monte et les sociétés abakua, furent discriminées. Des sanctions complémentaires étaient prévues par la loi à l'encontre de tout individu professant des religions " afro ", considérées comme relevant de la sorcellerie.

Les premières années de la période révolutionnaire virent se produire d'importants affrontements entre le nouvel État et les Églises chrétiennes, mettant en jeu un ensemble d'actions fort variées, mais possédant toutes un contenu nettement politique. Parmi les différents facteurs qui se trouvaient alors en jeu, citons la mentalité qui régnait en ces années de guerre froide, le fait que les dirigeants des cultes aient été pour la plupart étrangers, un certain élitisme dans la tradition de diverses églises dépendant à leur tour de structures étrangères, le manque d'ouverture qui caractérisait non seulement le catholicisme avant la tenue du Concile Vatican II, mais aussi les Églises évangéliques avant la célébration du Conseil Mondial des Églises. Par ailleurs, c'est un fait que la Révolution cubaine vint interrompre, en sauvegardant la laïcité constitutionnelle, un processus de réactivation du phénomène religieux qui était en train de se produire à la fin des années cinquante; l'Église catholique, qui manifesta le plus d'opposition à 
ce nouvel état des choses, se vit dès lors privée de sa position privilégiée et hégémonique.

En 1965, le Parti Communiste se constitua en tant que force politique dominante du pays ; ce parti rassembla les différents éléments qui étaient intervenus dans les luttes insurrectionnelles et les articula en une institution plus structurée et selon la conception idéologique du marxisme-léninisme, en vue d'un objectif politique central : la construction du socialisme. On considéra dès lors que ses militants avaient le devoir, entériné dans les statuts du parti, de «lutter contre l'obscurantisme religieux ", ce qui fut interprété de façon générale comme la nécessité d'exclure quiconque était croyant. Peu à peu, le modèle soviétique fut assimilé, avec sa conception de l'athéisme qualifié à tort de scientifique sur la base de critères étroits, dogmatiques et antidialectiques qui ne faisaient que nier les principes philosophiques sur lesquels était censée reposer la pratique politique. Cette situation se prolongea jusqu'à la fin des années quatre-vingts, tandis que la décennie suivante fut marquée par une conception plus objective et une pratique plus conséquente.

Tout un ensemble de phénomènes contribuèrent à réduire la présence de la religion dans la société au début des années soixante : la prise de position politique des Églises qui, comme nous l'avons vu, se traduisit par un moindre soutien populaire ; le départ de nombreux prêtres et pasteurs ; l'intensification du processus de sécularisation entamé auparavant ; les perspectives mondaines qui s'ouvraient alors à la satisfaction de demandes populaires jouissant d'un large soutien et, par voie de conséquence, l'abandon temporaire ou partiel du recours au surnaturel pour résoudre les problèmes et les difficultés, pratique courante au sein de la religiosité prédominante, comme nous l'avons déjà signalé. Par la suite, les prises de position en faveur de l'athéisme intensifieront encore ces tendances parmi la population, bien qu'il ne se soit jamais produit une conversion massive à la non-croyance.

\section{Compromis et évolution dans le paysage religieux cubain}

Dès la fin des années soixante, l'Église catholique s'ouvrit au dialogue, voire accepta certaines valeurs ayant cours dans la nouvelle société, comme il ressort des documents finaux de la Rencontre Nationale Ecclésiale Cubaine (ENEC) tenue en 1986 : ce fut à cette occasion que l'Église définit, de la façon la plus claire, sa position vis-à-vis du processus socialiste cubain, allant même jusqu'à reconnaître que la Révolution avait appris à donner par justice ce qu'on donnait auparavant par charité. Par la suite, il se produisit un certain nombre de changements ou d'alternances, mais toujours dans la ligne de la doctrine sociale de l'Église ; ces changements portaient tantôt sur de simples nuances, tantôt sur des questions plus profondes, notamment lorsque l'Église adopta une politique 
d'opposition lors des épisodes les plus difficiles de la crise économique des années quatre-vingt-dix.

En ce qui concerne les Églises protestantes, leur panorama sociopolitique se caractérisait par une importante hétérogénéité, aussi bien d'une Église à l'autre qu'au sein de chacune d'entre elles. Certaines déclarèrent ouvertement la guerre au processus révolutionnaire, surtout durant la première moitié des années soixante. Le non-respect de certains accords formels de la part des Témoins de Jéhovah, dans un climat de franche hostilité, entraîna la suppression de leur reconnaissance officielle auprès du Registre des Associations du ministère de la Justice pendant les années soixante-dix, tandis que leurs Salles du Royaume étaient affectées à des fonctions sociales (écoles, garderies d'enfants et autres services au bénéfice de la communauté locale).

Peu à peu ces attitudes se modérèrent ou se modifièrent considérablement. Certaines de ces Églises, qui avaient eu des réactions violentes face au changement social, organisèrent par la suite des camps de travail volontaire dans l'agriculture, réalisèrent des œuvres d'utilité communautaire ou participèrent à d'autres activités à caractère social. Ces derniers temps, les Témoins de Jéhovah ont manifesté leur ouverture au dialogue ainsi que leur volonté de demeurer à l'écart de toute action relevant d'une politique d'opposition.

C'est essentiellement au sein des Églises historiques, mais également des Églises pentecôtistes ${ }^{21}$ et d'autres encore répondant à des dénominations diverses, ainsi qu'à l'intérieur du mouvement œcuménique, que surgit très tôt une pensée théologique contextualisée, d'orientation sociale, engagée dans un projet social visant au progrès du peuple. Cette pensée coïncide dans son essence avec la théologie de la libération latino-américaine. Elle se distingue de celle-ci en ce qu'elle s'inscrit dans un contexte social différent, en ce que ses promoteurs sont définitivement favorables à l'option socialiste et en ce qu'elle est née avant son équivalent continental. L'analysant au niveau de son origine et de son contenu, Juana Berges et René Cárdenas l'ont désignée sous le nom de Nouvelle Théologie Cubaine ${ }^{22}$.

Les expressions religieuses qui se sont répandues parmi les secteurs populaires n'ont pas présenté de mouvements d'opposition organisée au processus révolutionnaire. Dans la plupart des cas, leurs représentants ont réagi en fonction

21. Il est significatif qu'à Cuba le pentecôtisme ne soit pas exclusivement synonyme de fuite dichotomique par rapport aux problèmes sociaux. Certains pentecôtistes - aussi bien des individus que des groupes, voire des Églises tout entières, comme l'Église Chrétienne Pentecôtiste - sont en accord avec certains objectifs du projet socialiste cubain et font partie du mouvement œcuménique.

22. Juana Berges, René Cárdenas, «El pastorado protestante y la Nueva Teología Cubana ", in La religión. Estudio de especialistas cubanos sobre la temática religiosa, La Habana, Edit. Política, 1993. 
d'aspirations et d'autres facteurs étrangers à toute théorie philosophique, politique et religieuse systématisée sous la forme d'une religion dotée de structures organisationnelles complexes. Tel est le cas des religions d'origine africaine, du spiritisme et de la religiosité populaire.

Malgré l'absence de toute intention délibérée et bien qu'elles soient soumises aux mêmes conditions d'ensemble favorisant l'athéisme, les religions d'origine africaine bénéficièrent d'un certain essor en raison de deux facteurs principaux : d'une part, le progrès social réel des populations modestes, parmi lesquelles se trouvaient la plupart des représentants religieux, et la création de conditions favorables à l'élimination des discriminations sociales et raciales; d'autre part, la politique de sauvegarde des racines africaines à travers des manifestations culturelles et folkloriques auxquelles ces expressions religieuses se trouvent intimement liées.

Malgré la mise en place de l'athéisme scientifique en tant que concept directeur, et surtout avant que celle-ci ne soit établie, la direction du Parti et de l'État s'attacha à définir peu à peu, depuis le moment de la prise du pouvoir et à partir de l'appel lancé auprès des croyants à participer à la "construction d'une nouvelle société ", quelques grandes lignes politiques reposant sur certaines idées centrales de la tradition cubaine : unité, droit de chacun à avoir des croyances religieuses et à les pratiquer, droit à ne pas être croyant et à ne pratiquer aucune religion (un droit qui représentait alors une innovation), égalité de toutes les religions (ce qui interdisait d'en privilégier ou, au contraire, d'en poursuivre une en particulier).

Les années quatre-vingt-dix furent marquées par une sévère crise économique que l'on a coutume de désigner sous le nom de "période spéciale »; celle-ci eut des répercussions sur bien d'autres domaines de la vie sociale, notamment en matière religieuse. Allant de pair avec la crise et étroitement lié à celle-ci, le regain d'activité que connut alors la religion entraîna, entre autres conséquences, une présence accrue de la religion dans la société cubaine - ce qui peut être perçu, notamment, à travers l'élargissement de l'espace social des organisations religieuses. Ainsi, les Églises chrétiennes dans leur ensemble ne disposent pas seulement d'un volume plus important de publications religieuses qu'au cours des années précédentes, mais le nombre de leurs fidèles, ainsi que celui de leurs dirigeants, s'est accru et elles ont droit désormais à de meilleures conditions pour mener à bien leurs activités de prosélytisme; en outre, elles participent à des projets de développement social et économique, d'aide humanitaire en faveur de collèges, d'hôpitaux, d'asiles et d'un certain nombre d'œuvres sociales, ce qui représente une reprise des activités d'assistance sociale chères à ces institutions.

De leur côté, en accord avec les correctifs qui sont alors apportés, les dirigeants politiques du pays prennent position en faveur d'une conception intégrale de la religion, allant au-delà de l'étroit athéisme scientifique et permettant une meilleure viabilité à l'application des grandes lignes qui orientent la politique 
officielle en la matière. En 1991 a lieu le IV Congrès du Parti, au cours duquel des accords importants sont adoptés. Ils comportent deux aspects dont les conséquences sont majeures pour l'activité religieuse : d'une part, la modification des statuts de manière à éliminer toute formulation pouvant être interprétée dans le sens de la nécessité d'exclure les croyants des listes du parti ; et d'autre part, l'adoption d'accords proposant des modifications du texte constitutionnel en vue de son perfectionnement, ce qui se produisit effectivement, grâce à la Réforme Constitutionnelle de 1992. Nous allons maintenant nous attacher à l'analyser en la comparant avec la Constitution socialiste de 1976, modifiée par cette réforme.

\section{La Réforme Constitutionnelle de 1992}

La Constitution socialiste approuvée lors du référendum de 1976, à travers le vote secret et direct de $98 \%$ de la population cubaine, croyants et non-croyants confondus, établissait le droit à la croyance, à la pratique religieuse ainsi que, pour la première fois, le droit à la non-croyance. Tout en conservant les antécédents constitutionnels, elle décrétait la séparation de l'Église, de l'État et de l'école. Ce texte n'instituait pas la morale chrétienne en tant que morale officielle. La loi, quant à elle, établissait que toute entrave à la liberté de culte était passible de sanction.

Aux termes de l'article 54, alinéa 3, de la Constitution de 1976, «il est illégal et punissable d'opposer la foi ou la croyance religieuse à la Révolution, à l'éducation ou à l'accomplissement du devoir de travailler, de défendre la patrie par les armes, de revendiquer ses symboles et tout autre devoir que prévoit la Constitution ". En 1992, la réforme de la Grande Charte supprima cet article. Dans son chapitre V sur l'égalité, la Constitution de 1976 ne faisait pas allusion à la non-discrimination pour des raisons religieuses, étant donné qu'en vertu de l'article 41 , alinéa $1^{\mathrm{er}}$, la loi interdisait et sanctionnait toute discrimination pour des raisons de race, de couleur, de sexe ou d'origine nationale. L'argument qui fut alors avancé prétendait qu'une telle prescription était inutile, dans la mesure où ceci n'était pas censé se produire dans la pratique sociale. Il fallut attendre la Réforme Constitutionnelle de 1992, approuvée par l'Assemblée Nationale du Pouvoir Populaire, pour que, à la suite de longues discussions et propositions, soient enfin offertes aux croyants les garanties légales les protégeant de toute éventualité ou tentative de marginalisation ou de discrimination de la part de personnes, d'institutions ou d'organismes gouvernementaux. L'article 42, inclus dans le chapitre VI consacré à l'égalité, dispose que « la discrimination pour des raisons de race, de couleur de peau, de sexe, d'origine nationale, de croyances religieuses ou pour toute autre raison portant atteinte à la dignité humaine, est interdite et sanctionnée par la loi ». L'article 43 vient compléter cette disposition en stipulant que les citoyens qui professent des croyances religieuses sont égaux aux autres devant la loi pour accéder, en fonction de leurs mérites et de leurs 
capacités, à tous les postes et emplois de l'État, de l'administration publique et de la prestation de services : suit une liste comprenant les services de santé, l'enseignement, les salaires, etc.

Faisant partie du chapitre VI, consacré aux droits, devoirs et garanties fondamentales, l'article 54 de la Grande Charte de 1976 fut modifié par la Réforme Constitutionnelle de 1992 (où il correspond à l'article 55). La version de 1976 établissait que l'État socialiste base son activité et éduque le peuple en accord avec la conception scientifique matérialiste de l'univers, ce qui est supprimé dans celle de 1992. Ce même article de la Constitution de 1976 déclarait que l'État reconnaît et garantit la liberté de conscience (celle de 1992 complète : «reconnaît, respecte et garantit la liberté de conscience et de religion»), le droit de chacun à professer n'importe quelle croyance religieuse et à pratiquer, dans le respect de la loi, le culte de son choix. La Constitution de 1992 réaffirme que l'État «reconnaît, respecte et garantit » la liberté de chaque citoyen, aussi bien de changer de croyances religieuses, que de n'en avoir aucune.

Enfin le chapitre I, "Fondements politiques, sociaux et économiques de l'État ", stipule, dans son article 8, que "l'État reconnaît, respecte et garantit la liberté religieuse. Dans la République cubaine, les institutions religieuses sont séparées de l'État. Les différentes croyances et religions jouissent toutes de la même considération ». De même, la loi continue à proscrire toute discrimination dans l'exercice du culte et, aux termes de l'article 294.1 et 2 du code pénal, établit comme circonstance aggravante le fait qu'un tel délit soit commis par un fonctionnaire ${ }^{23}$.

Ces modifications sont liées à un processus de changement au niveau de la conscience sociale, permettant dans l'ensemble une appréhension plus objective de la religion, de l'ensemble des rapports au sein desquels celle-ci est inscrite ainsi que du rôle social des organisations religieuses. Comme on le devine aisément, ce processus, loin d'être homogène et rapide, se heurte à la résistance des divers niveaux de la société, à des schémas préconçus, à des stéréotypes et préjugés de toute sorte, ce qui lui confère une évolution inégale. En même temps, il suscite des plaintes de la part des ecclésiastiques, ce qui entraîne des conflits plus ou moins graves entre l'Église et l'État, bien que les problèmes fondamentaux semblent avoir été résolus.

Si la Constitution n'affirme pas textuellement que l'État cubain est laïque, l'ensemble des articles dont elle se compose permet de l'interpréter dans ce sens. Fernández Bulté en propose l'analyse à partir de la «considération doctrinale »

23. La loi 62 du code pénal, entrée en vigueur le 30 avril 1988 et venant modifier la loi précédente, sanctionne dans son article 294, alinéas 1 et 2 (Délits contre la liberté de cultes), toute entrave à l'exercice de la liberté de culte au préjudice de quelque organisation religieuse reconnue que ce soit. 
de l'article 1, qui définit la nature, la forme républicaine unitaire et démocratique, ainsi que les objectifs essentiels de l'État; selon cet article, la souveraineté réside dans le peuple - ce qui, de toute évidence, signifie que la source du pouvoir n'est pas extérieure au monde -, tandis que l'article 8 détermine ce qui est essentiel au système politique, lorsqu'il déclare que celui-ci, de même que « l’État en tant que chaînon principal, n'a rien à voir avec aucune institution religieuse et, par conséquent, qu'aucune de ces institutions ou qu'aucune Église ne fait partie du système politique de la société cubaine ${ }^{24}$.

Il va de soi qu'une définition plus spécifique sur le plan des effets pratiques relève d'une législation complémentaire, notamment de la loi dite des cultes, annoncée par la Constitution. Toutefois, pour des raisons diverses, celle-ci est demeurée en suspens, en particulier depuis la dernière version de la loi de 1974 sur les associations, dont les clauses transitoires ont spécifié que les normes concernant les organisations religieuses devraient être établies par la loi des cultes. Sa promulgation immédiate ou son ajournement dans l'attente d'un moment plus propice ont donné lieu à divers débats. La complexité des enjeux d'une telle législation, mais également la nécessité de son existence en tant qu'instrument régulateur, tel que celui-ci existe de nos jours dans n'importe quel autre pays du monde, n'échappent à personne. Cependant, il faut tenir compte des pressions qu'exercent sur la vie politique cubaine des propagandes hostiles, qui veulent sans cesse voir une intervention de l'État dans la vie religieuse, tout en méconnaissant la pratique usuelle dans le domaine international.

Les antécédents historiques de la législation concernant le registre des associations religieuses sont particulièrement complexes. La République inaugurée en 1902 hérita d'une législation qui obligeait toute organisation religieuse à se faire enregistrer ; toutefois, l'Église catholique échappait à cette obligation. Pendant la période révolutionnaire, le Registre des Associations, rattaché tout d'abord au ministère de l'Intérieur, puis à celui de la Justice, se chargea de contrôler les organisations enregistrées, tout en excluant l’Église catholique, et sans créer de mécanismes ad hoc prenant en compte les groupements relevant des religions d'origine africaine. Certaines associations catholiques furent néanmoins enregistrées, ainsi que des confréries et des instituts. Des centres spirites n'ayant pas renouvelé leur inscription lorsqu'il leur était demandé de le faire, ou ne s'étant jamais inscrits, continuent à fonctionner sans aucun contrôle ou doivent demander une autorisation pour célébrer certaines de leurs cérémonies, ce qui est également le cas pour certaines manifestations d'origine africaine. Par ailleurs, la loi avait toujours été arbitraire tout au long de la république néocoloniale, dans la mesure où elle considérait que les pratiques d'origine africaine représentaient des circonstances aggravantes en cas de délit. La Révolution abrogea cette législation et, désireuse de consacrer le droit à la liberté religieuse, définit le délit d'entrave à cette liberté.

24. Dr. Julio Fernández Bulté, communication présentée au CIPS (voir note 20). 
De fait, si les bases juridiques du fonctionnement conséquent d'un État laïque ont bien été jetées, le processus correspondant n'est pas encore achevé.

\section{Quelques considérations finales}

Les attitudes vis-à-vis du laïcisme sont contradictoires. D'une part, certains défendent l'idée selon laquelle la liberté de religion implique la possibilité, pour les institutions religieuses, d'intervenir activement dans le processus social. Cette attitude, associée à la direction des organisations religieuses, privilégie en général une religion particulière. Mais un autre point de vue, propre aux mouvements religieux progressistes, considère que les Églises n'ont pas à remplir les fonctions propres à l'État, même si le pouvoir politique doit garantir le droit à adopter des croyances et à les pratiquer. Ces différends correspondent à des prises de position antagonistes concernant l'origine du pouvoir. Pour les uns, il appartient à la religion de structurer la société, et il importe qu'il existe une garantie en ce sens. Pour les autres, et en accord avec un laïcisme conséquent, l'État doit, et peut, disposer de fonctions d'intégration sociale, dans la mesure où le pouvoir émane de la société elle-même et non d'une source externe, comme pourrait l'être le surnaturel.

Au sein de ces polémiques, un enjeu important concerne le caractère public ou privé de la religion. L'expérience montre qu’il ne saurait être question de nier la dimension sociale propre à la religion, non seulement parce que celle-ci constitue en elle-même un phénomène social, mais aussi parce que ses adeptes sont des êtres sociaux de par leur nature humaine, au même titre que les non-croyants. Le problème se situe à la limite entre le domaine public et les prétentions politiques des organisations religieuses et pose qu'il n'est pas opportun que la religion participe du pouvoir politique en tant qu'élément de légitimation d'un système ou d'un régime déterminé, voire d'un parti spécifique, ni qu'une institution religieuse quelconque soit habilitée à prendre des décisions en matière politique, ou soit privilégiée par l'État, cela afin d'éviter toute discrimination vis-à-vis d'autres institutions et croyances religieuses.

Malgré son profond engagement envers le régime colonial, l'Église continue à bénéficier, dans l'ensemble des pays latino-américains, d'une situation privilégiée ainsi que d'une part considérable de pouvoir et d'influence politiques. Ajoutons que huit pays latino-américains au moins méritent d'être qualifiés d'États confessionnels, malgré certaines nuances et, bien entendu, en raison de la place privilégiée qu'occupe l'Église catholique dans la plupart d'entre eux, comme c'était le cas à Cuba à l'époque prérévolutionnaire. Cependant, tous reconnaissent au moins la liberté de culte, avec certaines limitations parfois. Reste à savoir jusqu'à quel point celle-ci est effectivement respectée dans la pratique politique quotidienne.

Dans d'autres pays du monde, on constate également une grande diversité des définitions adoptées par les Constitutions en vigueur. Dans certains cas le 
caractère confessionnel de l'État peut être reconnu, quoique selon des modalités diverses. Si dans certains pays cela ne signifie nullement que la pluralité religieuse est interdite ou n'est pas reconnue, dans d'autres au contraire - comme c'est le cas dans certains pays islamiques - l'identification de la nation à une religion déterminée ne laisse guère de place, voire aucune, à d'autres formes de religion ni, à plus forte raison, à la non-croyance. Ceci est source de violence entre institutions ou entre citoyens de différentes confessions, voire au sein de l'État lui-même.

Mais la sphère politique n'est pas la seule à ne pas reconnaître la liberté de religion. Diverses institutions faisant partie de ce qu'il est convenu d'appeler les nouveaux mouvements religieux (un concept possédant certaines caractéristiques que les recherches en la matière doivent mettre en évidence, bien que l'on ait au moins une idée de leurs formes et de leurs regroupements les plus significatifs, ainsi que de leur manière d'agir) ont coutume d'utiliser leurs moyens de communication, ainsi que les prêches prononcés à l'occasion des cérémonies de culte, pour se livrer à un prosélytisme qui ne vise pas tant à divulguer leurs doctrines et leurs principes qu’à jeter le discrédit sur d'autres religions, avec des accents parfois franchement agressifs. Il arrive même que certaines institutions religieuses s'en prennent au laïcisme ou jettent l'anathème contre les non-croyants, méconnaissant le droit sur lequel se fonde un tel choix.

Certains milieux religieux alimentent des désaccords, souvent historiques, entre différentes religions ou institutions religieuses, ainsi que des points de vue profondément discriminatoires, reposant sur des préjugés envers d'autres religions ou d'autres croyances et pratiques ne faisant pas partie d'orthodoxies reconnues : tel est le cas, assez fréquent à Cuba, de certains chrétiens vis-à-vis des religions d'origine africaine, ou encore de certaines hiérarchies ecclésiastiques qui considèrent la religiosité populaire, fort répandue en Amérique latine et prédominante à Cuba, comme relevant d'une foi incomplète, ou représentant même la manifestation de simples superstitions.

La Constitution de plusieurs pays, y compris des pays latino-américains, ou leurs lois particulières établissent des interdictions ou des normes restrictives. C'est le cas des nations qui interdisent aux ministres du culte (prêtres, pasteurs ou autres dignitaires et représentants de la hiérarchie religieuse) d'occuper des postes publics tels que ceux de président ou de vice-président de la nation, de membre du congrès, de député ou de ministre, ou même de présenter leur candidature à de tels postes; c'est également le cas des pays qui interdisent aux partis confessionnels de participer aux campagnes électorales.

Plusieurs pays connaissent des difficultés quant à la manière dont l'État traite des problèmes religieux. Parfois, il n'existe pas même un organisme chargé de la question, alors que dans d'autres cas cet organisme est inefficace, voire totalement inopérant. Dans d'autres cas encore, les plaintes sont dues à la place de cet organisme à l'intérieur de l'appareil étatique, dans des structures chargées de 
questions internes et policières, si bien que sa fonction est essentiellement de l'ordre du contrôle et, en un certain sens, de la répression.

D'un autre point de vue, on ne saurait oublier que tout État, qu'il soit laïque ou confessionnel, ne peut en aucun cas renoncer à sa fonction de sauvegarde des intérêts de tous. Or ceci s'avère particulièrement difficile pour l'État cubain dans les circonstances actuelles, vu le harcèlement auquel il est soumis depuis l'extérieur et la situation économique complexe qu'il traverse. Ce qui est en jeu, c'est l'affaiblissement de la pratique laïque que l'on constate dans le monde, et le renforcement des pressions provenant d'institutions religieuses. Celles-ci s'appuient, en général, sur l'essor du phénomène religieux auquel on assiste à l'échelle mondiale, en vue de récupérer leurs positions au sein du pouvoir et, dans certains cas particuliers, en vue de revenir à leur ancienne position hégémonique. Ceci est particulièrement évident dans les revendications de l'Église catholique en Amérique latine, y compris à Cuba. Dans certaines régions, on constate des prises de position de la part de gouvernements qui ont intérêt à être légitimées par des bases religieuses, ou à obtenir le soutien d'une institution religieuse en particulier.

Un inventaire minimal des limitations, voire des violations que subit la liberté de religion, de croyance et de culte, en tant qu'expression des droits de l'homme, aussi bien au niveau de la législation que de la pratique sociale, devrait comprendre les quelques points suivants :

- la déclaration d'États confessionnels;

- les définitions légales et pratiques bénéficiant à une religion ou une institution religieuse, au détriment des autres;

- la non-reconnaissance de la non-croyance et de la pratique du culte en tant que droits des personnes;

- les serments prêtés sur des textes religieux lors des dépositions en justice;

- la persistance de luttes entre religions et de préjugés concernant certaines d'entre elles ;

- l'existence de fondamentalismes religieux ;

- les discriminations pour des raisons de xénophobie, y compris vis-à-vis de la religion des immigrés;

- l'utilisation d'arguments basés sur des contradictions entre religions, afin de justifier des agressions obéissant à des intérêts politiques et économiques;

- la défense de l'enseignement religieux dans les écoles publiques, impliquant la préférence accordée à une religion déterminée, face à l'impossibilité pratique de les inclure toutes dans l'enseignement, et violation du droit à la non-croyance dans les cas où cet enseignement est obligatoire;

- l'importance démesurée accordée au droit de croyance et de pratique religieuse par rapport aux droits véritablement fondamentaux;

- la surestimation de l'influence réelle d'une religion sur la culture et l'histoire des nations ; 
- les initiatives des représentants de la hiérarchie religieuse en vue de récupérer leurs anciennes positions et de permettre à leurs institutions d'intervenir au niveau des décisions politiques, ou d'accroître leur influence sociale, éventuellement jusqu’à atteindre des niveaux hégémoniques ;

- la critique réitérée du laïcisme et des processus de sécularisation qui se sont produits au cours de l'histoire de l'humanité ;

- les tentatives de mettre en place une globalisation visant à imposer des formes de religion répondant aux intérêts des pays puissants, au détriment des traditions locales.

L'édification et l'amélioration constante d'une société, dans laquelle l'État se porte pleinement garant de l'exercice de la liberté religieuse, sans interférence d'aucune sorte avec des intérêts étrangers au bien commun, peuvent et doivent être le fait d'hommes et de femmes de bonne volonté, indépendamment du fait que ceux-ci croient en telle ou telle manifestation religieuse, ou qu'ils ne croient en aucune.

Jorge Ramírez CALZADILLA (†)

\section{Résumé}

L'auteur propose d'abord quelques définitions du mot "laïc " et analyse certaines des problématiques actuelles dont il fait l'objet. Pour lui, la laïcité s'incarne dans deux éléments : la liberté de religion et l'État lä̈que. Dans cette perspective, les principes qui définissent l'État lä̈que comportent autant de droits et de devoirs pour l'État que pour les organisations religieuses. L'article se concentre ensuite sur l'exemple cubain. Il y est présenté dans sa spécificité par rapport aux autres pays de l'Amérique latine. L'étape coloniale - de la Conquête à la colonisation espagnole, puis la période républicaine néocoloniale allant de 1902 à 1959 sont abordées, mais l'analyse est centrée sur l'étape révolutionnaire ultérieure. La conclusion tente d'inscrire la situation cubaine dans le contexte plus large des problématiques qui traversent les rapports entre religion et pouvoir politique dans le monde.

Mots-clés : Cuba, liberté de religion, État lä̈que, sécularisation.

\section{Abstract}

On the one hand, this article proposes some definitions of the term "secularism" and analyses different contemporary debates about secularism in Cuba. The author proposes to focus on the two main principles of secularism, that is to say the freedom of religion, equality and the secular state. These two principles imply both duties and rights for the state and for the religious groups. On the second hand, this article 


\section{82 - ARCHIVES DE SCIENCES SOCIALES DES RELIGIONS}

presents the specific case of secularism in Cuba. After having given a view of the historical context, the article focuses on the revolutionary period and proposes an analysis of the relation between church and state in Cuba in the light of different models of secularism in the world.

Key words: Cuba, freedom of religion, secular State, secularization.

\section{Resumen}

El presente artículo intenta proponer algunas definiciones de lo laico y analizar problemáticas presentes en la actualidad, particularizando en la realidad cubana, que es el objetivo central. Así se examina la noción de "laico", enfatizando en sus diferentes concepciones, así como en su distinción con respecto a otros conceptos que le son próximos, como el de "secular" y el de "ateo". Enseguida, se examinan los dos elementos en que se concreta el laicismo, a saber, la libertad de religión, y el Estado laico. En particular, se analizan algunas de las premisas que determinan al Estado laico, mismas que comportan tanto derechos como deberes, lo mismo para el Estado que para las organizaciones religiosas. Se enfatiza en el caso cubano desde su particularidad con respecto al resto de los países latinoamericanos. Aunque se mencionan algunos elementos de sus dos primeros periodos (la etapa colonial y el periodo de la república neocolonial), el análisis se centra, sobre todo, en la etapa revolucionaria posterior a dicha fecha. Para concluir, se intenta inscribir a la situación cubana en el contexto más amplio de las problemáticas que atraviesan a las relaciones entre la religión y el poder político en otras partes del mundo.

Palabras clave: Cuba, libertad de religión, Estado laico, secularización. 